\title{
Chronic Intestinal Pseudo-obstruction and Orthostatic Hypotension Associated with Small Cell Lung Cancer that Improved with Tumor Reduction after Chemoradiotherapy
}

\author{
Yusuke Izumi, Takeshi Masuda, Yasushi Horimasu, Taku Nakashima, Shintaro Miyamoto, \\ Hiroshi Iwamoto, Kazunori Fujitaka, Hironobu Hamada and Noboru Hattori
}

\begin{abstract}
:
Chronic intestinal pseudo-obstruction (CIPO) is a rare disease with symptoms of ileus without obstruction. Most cases of CIPO are idiopathic, and CIPO as a paraneoplastic neurological syndrome (PNS) associated with small cell lung cancer (SCLC) is rare. A 63-year-old man was diagnosed with functional ileus and confined to bed due to orthostatic hypotension. Chest computed tomography revealed a right hilar mass suspected of being lung cancer. Based on detailed examinations, he was diagnosed with limited-stage SCLC. His symptoms were confirmed as PNS because his serum anti-Hu antibody was positive. His PNS was improved with complete tumor reduction by chemoradiotherapy.
\end{abstract}

Key words: chronic intestinal pseudo-obstruction, small cell lung cancer, paraneoplastic neurological syndrome, orthostatic hypotension, anti-Hu antibody

(Intern Med 56: 2627-2631, 2017)

(DOI: 10.2169/internalmedicine.8574-16)

\section{Introduction}

Chronic intestinal pseudo-obstruction (CIPO) is a rare digestive disease with clinical symptoms of ileus due to severe gastrointestinal dysmotility without mechanical obstruction. Most cases of CIPO are idiopathic, and CIPO as a paraneoplastic neurological syndrome (PNS) associated with small cell lung cancer (SCLC) is rare (1). In addition, whether or not paraneoplastic CIPO can be resolved by tumor reduction is unclear. In this report, we present a case of paraneoplastic CIPO and orthostatic hypotension $(\mathrm{OH})$ associated with SCLC that was improved with a complete response of the tumor to chemoradiotherapy (CRT).

\section{Case Report}

A 63-year-old Japanese man presented to a local hospital because of anorexia and vomiting from June 2014. Abdominal computed tomography (CT) revealed intestinal dilatation indicating ileus. Abdominal surgery did not reveal any me- chanical intestinal obstruction. Based on this finding, he was diagnosed with functional ileus. Furthermore, the ileus was considered PNS because a 30 -mm right hilar mass suspected of being lung cancer was also detected on chest CT. Therefore, he was transferred to our hospital in the first week of October 2014.

$\mathrm{He}$ formerly smoked 20 cigarettes a day for 33 years. He had lost 20 kilograms of body weight (from 60 to $40 \mathrm{~kg}$ ) over the previous 3 months and needed intravenous hyperalimentation because he was unable to take meals. An abdominal examination revealed his bowel sounds were hardly audible. In addition, he had severe $\mathrm{OH}$, with his systolic blood pressure decreasing with presyncope from $120 \mathrm{mmHg}$ in the supine position to $50 \mathrm{mmHg}$ in the sitting position. There were no specific neurological findings other than those of ileus and $\mathrm{OH}$. His cranial nerve functions were intact, and no signs of cerebellar disease were elicited. His muscle strength, sensation, and deep tendon reflexes were intact on all extremities.

Laboratory tests revealed moderate anemia, hyponatremia, hypoalbuminemia, and slight elevation of the progastrin- 
Table. Laboratory Findings on Admission.

\begin{tabular}{lclllr}
\hline Hematology & & \multicolumn{2}{l}{ Biochemistory } & \multicolumn{2}{l}{ Tumor markers } \\
WBC & $9,730 / \mu \mathrm{L}$ & AST & $7 \mathrm{U} / \mathrm{L}$ & CEA & $2.6 \mathrm{ng} / \mathrm{mL}$ \\
Neut & $69.8 \%$ & ALT & $6 \mathrm{U} / \mathrm{L}$ & CYFRA & $1.7 \mathrm{ng} / \mathrm{mL}$ \\
Lym & $23.6 \%$ & LDH & $110 \mathrm{U} / \mathrm{L}$ & ProGRP & $154.4 \mathrm{pg} / \mathrm{mL}$ \\
Mono & $6.3 \%$ & ALP & $136 \mathrm{U} / \mathrm{L}$ & NSE & $11.3 \mathrm{ng} / \mathrm{mL}$ \\
Eos & $0.1 \%$ & T-Bil & $0.7 \mathrm{mg} / \mathrm{dL}$ & & \\
Baso & $0.2 \%$ & Na & $130 \mathrm{mEq} / \mathrm{L}$ & Anti-neuronal antibodies \\
RBC & $321 \times 10^{4} / \mu \mathrm{L}$ & $\mathrm{K}$ & $3.9 \mathrm{mEq} / \mathrm{L}$ & Hu & $(+)$ \\
Hgb & $9.1 \mathrm{~g} / \mathrm{dL}$ & Cl & $93 \mathrm{mEq} / \mathrm{L}$ & Yo & $(-)$ \\
Hct & $28.2 \%$ & TP & $5.6 \mathrm{~g} / \mathrm{dL}$ & Ri & $(-)$ \\
PLT & $48.2 \times 10^{4} / \mu \mathrm{L}$ & Alb & $2.6 \mathrm{~g} / \mathrm{dL}$ & CV2 & $(-)$ \\
& & BUN & $6.3 \mathrm{mg} / \mathrm{dL}$ & Ma1 & $(-)$ \\
Serology & & Cr & $0.56 \mathrm{mg} / \mathrm{dL}$ & Ma2 & $(-)$ \\
CRP & $6.2 \mathrm{mg} / \mathrm{dL}$ & & & Amphiphysin & $(-)$ \\
\hline
\end{tabular}

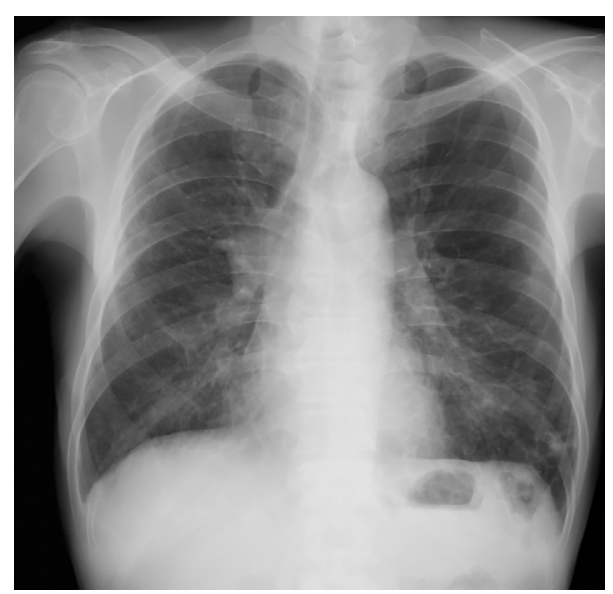

Figure 1. A chest radiograph taken at the patient's admission to our hospital showed swelling of the right hilum and scattered ground-glass opacity.

releasing peptide level (Table). Chest radiography revealed swelling of the right hilum and scattered ground-glass opacity, which suggested inflammatory changes after aspiration pneumonia due to vomiting (Fig. 1). Chest (Fig. 2) and abdominal CT scans (Fig. 3) showed findings similar to those of the previous CT scans obtained at the local hospital. Endobronchial ultrasound-guided transbronchial needle aspiration of the hilar mass revealed small cell carcinoma. ${ }^{18} \mathrm{~F}-$ fluorodeoxyglucose positron emission tomography (FDGPET) and cerebral magnetic resonance imaging revealed no metastatic lesions. From these observations, he was diagnosed with limited-stage SCLC, clinical T1b N1 M0 stage IIA. Furthermore, his functional ileus was diagnosed as CIPO because no organic disorder was detected on upper or lower gastrointestinal endoscopies or small bowel radiography. In addition, both CIPO and $\mathrm{OH}$ were diagnosed as PNS because anti-Hu antibody was detected in his serum sample and no underlying diseases, such as amyloidosis or diabetic neuropathy, which induce CIPO, were observed. Although he was not able to take meals, he suffered no gastrointestinal symptoms from being dependent on intravenous hypera- limentation. In addition, his condition, except for $\mathrm{OH}$ and slight anemia, made him eligible for CRT with cisplatin. Therefore, we decided to start CRT with the expectation that the antitumor effect would improve his PNS.

Accelerated hyperfractionated radiotherapy (54 Gy in total) and concurrent chemotherapy with cisplatin $\left(80 \mathrm{mg} / \mathrm{m}^{2}\right.$, day 1) and etoposide (100 mg/m $\mathrm{m}^{2}$, days $\left.1-3\right)$ were started. However, this regimen was changed to the following regimen from the second to the fourth course of chemotherapy because of febrile neutropenia: carboplatin (area under the curve of 5 , day 1) and etoposide $\left(80 \mathrm{mg} / \mathrm{m}^{2}\right.$, days $1-3$; Fig. 4). Two courses of chemotherapy and radiotherapy resulted in a complete response of the tumor (Fig. 5). The anti-Hu antibody test turned negative after CRT. Subsequently, prophylactic cranial irradiation (25 Gy in total) was performed. His symptoms of CIPO and $\mathrm{OH}$ gradually improved, and they completely disappeared six months after the initial treatment. No sign of recurrence was observed for 12 months after the initial treatment.

\section{Discussion}

We herein present a case of CIPO and $\mathrm{OH}$ associated with SCLC that were improved with complete tumor reduction after CRT. CIPO is characterized by the signs and symptoms of mechanical bowel obstruction without anatomic lesions. In a previous report of 121 CIPO cases, most cases were idiopathic (70.2\%), and the most frequent secondary cause was systemic sclerosis (16.6\%), followed by mitochondrial encephalomyopathy (5.2\%), amyloidosis (3.5\%), and hypothyroidism (2.6\%) (1). Cases of CIPO as PNS are rare. PNS, which is classified as "classical" and "non-classical", is an immune-mediated nervous system disorder that occurs in patients with cancer. Classical PNS comprises neurological symptoms that are often associated with cancer, including encephalomyelitis, limbic encephalitis, subacute cerebellar degeneration, opsoclonus-myoclonus, subacute sensory neuronopathy, CIPO, Lambert-Eaton myasthenic syndrome, and dermatomyositis. In contrast, non clas- 


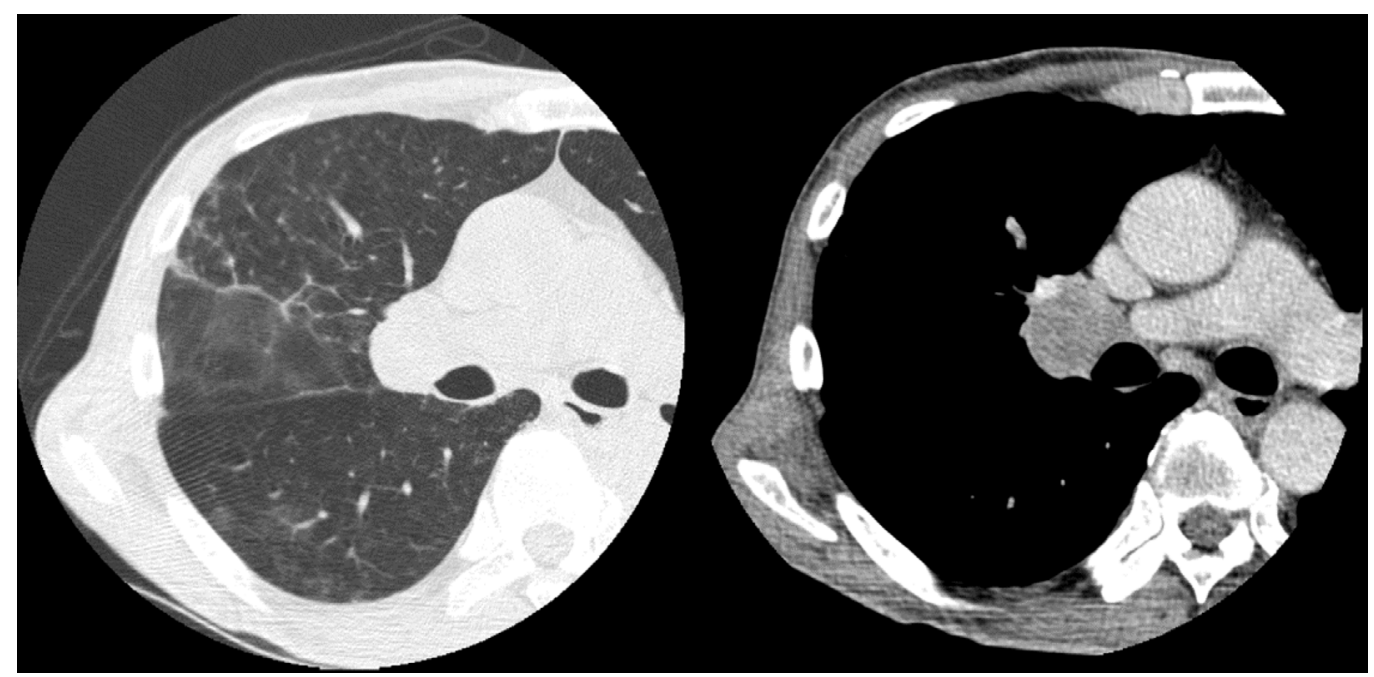

Figure 2. A chest CT scan on admission showed a 30-mm right hilar mass.

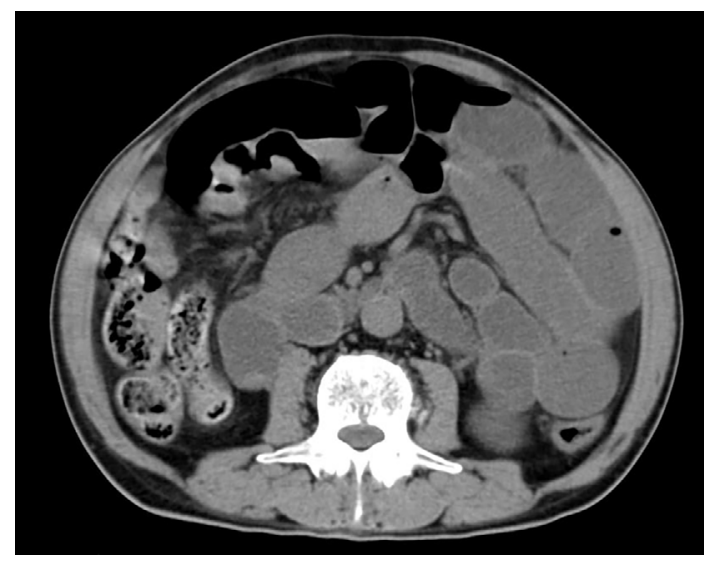

Figure 3. An abdominal CT scan on admission showed intestinal dilatation with air-fluid levels.

sical PNS comprises various neurological symptoms, including $\mathrm{OH}$, that can occur as PNS (2).

Some cases of PNS are associated with the presence of antibodies directed against antigens expressed in both cancer and nervous cells. Among the PNS antibodies, anti-Hu antibody is associated with sensory neuropathy, limbic encephalitis, and subacute cerebellar degeneration in various cancers (3). In a previous series of 132 SCLC patients with PNS and anti-Hu antibody, the most common neurological manifestation was neuropathy, which included peripheral neuropathy in $90 \%$ of patients, autonomic neuropathy in $18 \%$, and CIPO in 5\% (4). In contrast, anti-Hu antibody was detected in 10 of 12 SCLC patients with paraneoplastic gastrointestinal motor dysfunction (5-8). This observation suggests that anti-Hu antibody correlates with the etiology of paraneoplastic gastrointestinal motor dysfunction, including CIPO.

The present patient's Eastern cooperative oncology group performance status (PS) (9) was graded as 4 because of the presence of CIPO and $\mathrm{OH}$. Nevertheless, we were able to complete CRT. In addition, the PNS was improved, and a complete response of the tumor was achieved. Both early treatment and tumor reduction before neuronal death are generally thought to be required for the improvement of PNS (10). However, to our knowledge, only one case has been reported (by Mifune et al.) in which paraneoplastic CIPO associated with SCLC was improved after complete tumor remission by chemoradiotherapy (11). In that previous report, similar to our case, the onset of CIPO preceded the initiation of CRT by three months. However, Lee et al. reported that, in 9 cases of SCLC with paraneoplastic gastrointestinal motor dysfunction, the onset of gastrointestinal symptoms preceded the diagnosis of SCLC by a mean interval of 8.7 months (5). From these observations, we can speculate that the improvement of CIPO by anticancer treatment, as was attained in both our case and that reported by Mifune et al., was mainly due to the early diagnosis and complete tumor remission. Because no cases of paraneoplastic $\mathrm{OH}$ associated with SCLC that improved after tumor reduction have been reported, we cannot discuss why the $\mathrm{OH}$ improved in this case. However, the above-mentioned reasons would also apply to $\mathrm{OH}$. Therefore, the primary cancer in patients with paraneoplastic CIPO and/or $\mathrm{OH}$ should be diagnosed as early as possible, and anticancer treatment should be urgently initiated to improve the syndromes.

Limited-stage SCLC was completely improved in the present case by administering CRT, and no sign of recurrence was observed for 12 months after the initial treatment. With regard to the prognostic implications of anti-Hu antibody, Graus et al. reported that anti-Hu antibody positivity in SCLC patients was associated with limited staging, a complete response to therapy, and a longer survival (12). The progress or dissemination of cancer cells in the present study may have been suppressed by some immune reactions associated with anti-Hu antibody. Given these findings, even in SCLC patients with poor PS due to PNS, the induction of anticancer treatment should be considered because it might induce a good tumor response with improvement of PNS. 


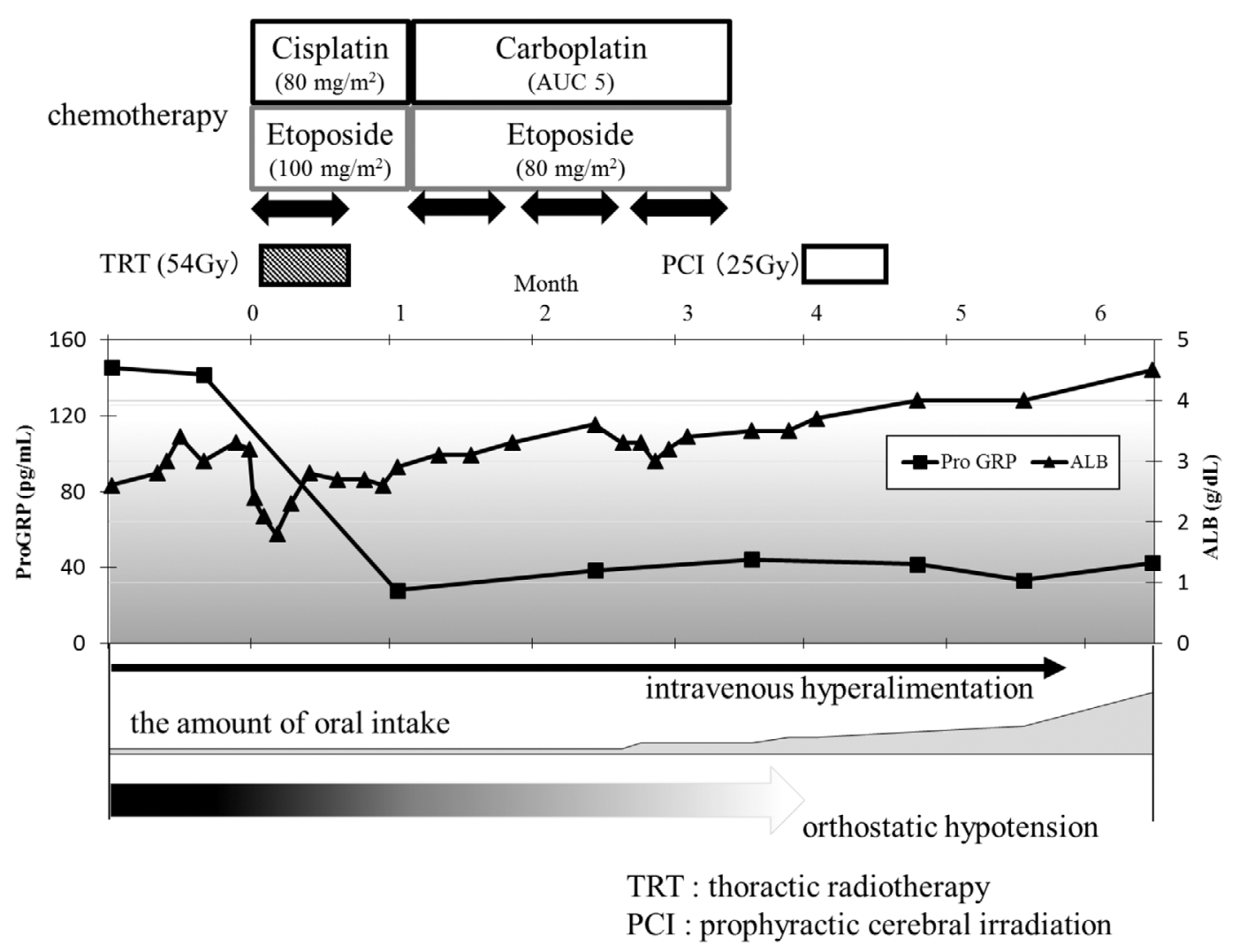

Figure 4. The treatment regimen and changes in the levels of tumor markers and serum albumin, orthostatic hypotension, and the amount of oral intake. The amount of oral intake increased gradually with tumor reduction, and the orthostatic hypotension was also improved.

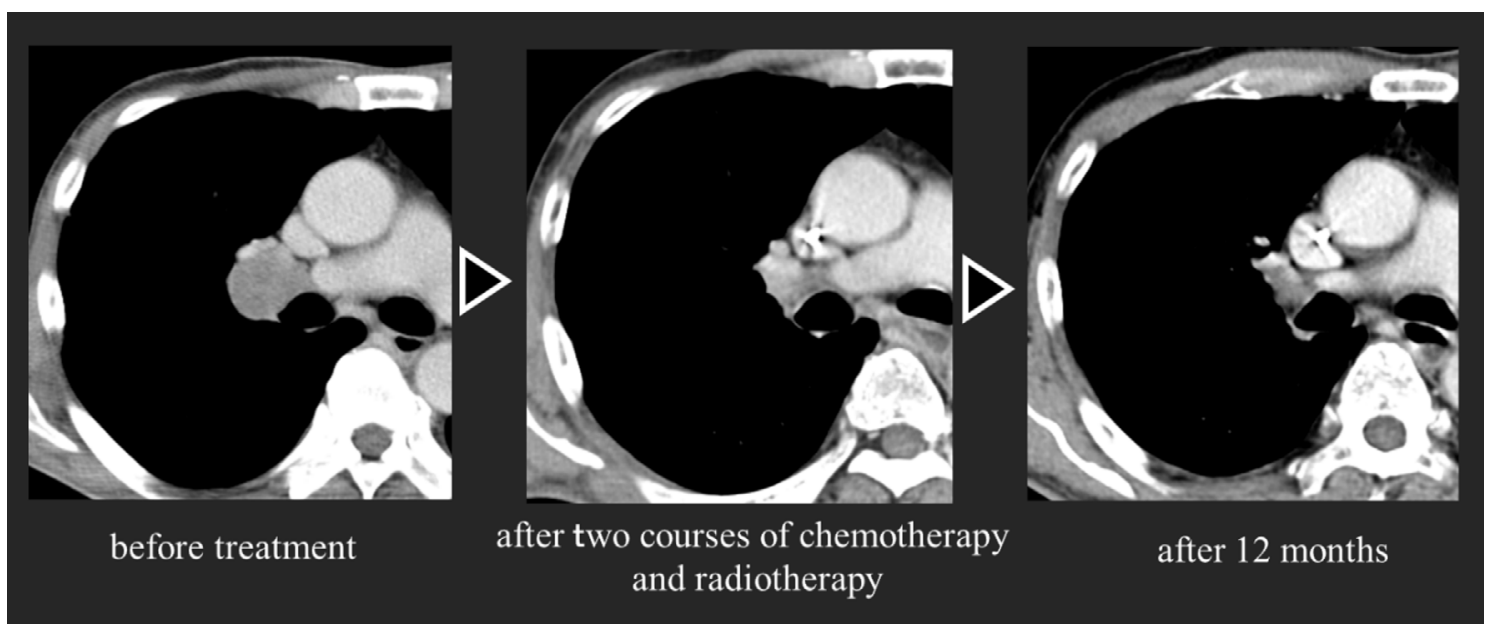

Figure 5. Two courses of chemotherapy and radiotherapy resulted in a complete response of the tumor. There has been no sign of recurrence for more than 12 months after the initial treatment.

\section{Conclusion}

We herein present a case of CIPO and $\mathrm{OH}$ associated with SCLC. In this case, CRT resulted in a complete response of the tumor and improvement of the CIPO and $\mathrm{OH}$. Although paraneoplastic CIPO is rare, the possibility of an underlying tumor in patients with functional ileus should be considered. In addition, we must urgently diagnose CIPOcausing primary cancer and initiate anticancer treatment be- fore irreversible neuronal damage occurs.

The authors state that they have no Conflict of Interest (COI).

\section{Acknowledgement}

We thank Drs. Ayumi Uchibori and Atsuro Chiba (Department of Neurology, School of Medicine, Kyorin University) for performing the anti-neuronal antibody testing. 


\section{References}

1. Iida $H$, Inamori $M$, Sekino $Y$, Sakamoto $Y$, Yamato S, Nakajima A A review of the reported cases of chronic intestinal pseudoobstruction in Japan and an investigation of proposed new diagnostic criteria. Clin J Gastroenterol 4: 141-146, 2011.

2. Graus F, Delattre JY, Antoine JC, et al. Recommended diagnostic criteria for paraneoplastic neurological syndromes. J Neurol Neurosurg Psychiatry 75: 1135-1140, 2004.

3. Vedeler CA, Antoine JC, Giometto B, et al. Management of paraneoplastic neurological syndromes: report of an EFNS Task Force. Eur J Neurol 13: 682-690, 2006.

4. Lucchinetti CF, Kimmel DW, Lennon VA. Paraneoplastic and oncologic profiles of patients seropositive for type 1 antineuronal nuclear autoantibodies. Neurology 50: 652-657, 1998.

5. Lee HR, Lennon VA, Camilleri M, Prather CM. Paraneoplastic gastrointestinal motor dysfunction: clinical and laboratory characteristics. Am J Gastroenterol 96: 373-379, 2001.

6. Taverna JA, Babiker HM, Yun S, et al. The great masquerader of malignancy: chronic intestinal pseudo-obstruction. Biomark Res 2: 23, 2014.

7. Moskovitz DN, Robb KV. Small cell lung cancer with positive anti-Hu antibodies presenting as gastroparesis. Can J Gastroenterol
16: $171-174,2002$

8. Sorhaug S, Steinshamn SL, Waldum HL. Octreotide treatment for paraneoplastic intestinal pseudo-obstruction complicating SCLC. Lung Cancer 48: 137-140, 2005.

9. Oken MM, Creech RH, Tormey DC, et al. Toxicity and response criteria of the Eastern Cooperative Oncology Group. Am J Clin Oncol 5: 649-655, 1982.

10. Yamamoto T, Tsuji S. Neurological syndromes, encephalitis. Gan To Kagaku Ryoho (Japanese Journal of Cancer and Chemotherapy) 37: 995-1005, 2010 (in Japanese, Abstract in English).

11. Mifune D, Tsukada H, Hosoi M, Okajima M, Yokoyama A. Chronic intestinal pseudo-obstruction as a paraneoplastic presentation of limited-stage small cell lung cancer. Nihon Kokyuki Gakkai Zasshi (Annals of The Japanese Respiratory Society) 48: 439443, 2010 (in Japanese, Abstract in English).

12. Graus F, Dalmou J, Reñé R, et al. Anti-Hu antibodies in patients with small-cell lung cancer: association with complete response to therapy and improved survival. J Clin Oncol 15: 2866-2872, 1997.

The Internal Medicine is an Open Access article distributed under the Creative Commons Attribution-NonCommercial-NoDerivatives 4.0 International License. To view the details of this license, please visit (https://creativecommons.org/licenses/ by-nc-nd/4.0/).

(C) 2017 The Japanese Society of Internal Medicine Intern Med 56: 2627-2631, 2017 